\title{
The zinc finger proteins ZNF644 and WIZ regulate the G9a/GLP complex for gene repression
}

\author{
Chunjing Bian ${ }^{\dagger}$, Qiang Chen ${ }^{\dagger}$, Xiaochun Yu* \\ Division of Molecular Medicine and Genetics, Department of Internal Medicine, \\ University of Michigan Medical School, Ann Arbor, United States
}

\begin{abstract}
The G9a/GLP complex mediates mono- and dimethylation of Lys9 of histone H3 at specific gene loci, which is associated with transcriptional repression. However, the molecular mechanism by which the G9a/GLP complex is targeted to the specific gene loci for $\mathrm{H} 3 \mathrm{~K} 9$ methylation is unclear. In this study, with unbiased protein affinity purification, we found ZNF644 and WIZ as two core subunits in the G9a/GLP complex. ZNF644 and WIZ interact with the transcription activation domain of G9a and GLP, respectively. Moreover, both ZNF644 and WIZ contain multiple zinc finger motifs that recognize consensus DNA sequences. ZNF644 and WIZ target G9a and GLP to the chromatin and mediate the G9a/GLP complex-dependent H3K9 methylation as well as gene repression. Thus, our studies reveal two key subunits in the G9a/GLP complex that regulate the function of this histone methyltransferase complex.
\end{abstract}

DOI: 10.7554/eLife.05606.001

*For correspondence: xiayu@ umich.edu

tThese authors contributed equally to this work

Competing interests: The authors declare that no competing interests exist.

Funding: See page 15

Received: 13 November 2014 Accepted: 26 February 2015 Published: 19 March 2015

Reviewing editor: Ali Shilatifard, Northwestern University Feinberg School of Medicine, United States

Copyright Bian et al. This article is distributed under the terms of the Creative Commons Attribution License, which permits unrestricted use and redistribution provided that the original author and source are credited.

\section{Introduction}

Post-translational modifications of histones, especially lysine methylation, play important roles in diverse biological processes, such as gene transcription, chromatin packaging, and cellular differentiation. Different lysine residues of histones are methylated by different histone lysinespecific methyltransferases (HKMTs). Among HKMTs, G9 and GLP specifically catalyze H3K9 mono(H3K9me1) and dimethylation (H3K9me2) (Tachibana et al., 2001, 2005; Kubicek et al., 2007).

G9a and GLP are paralogs with similar domain architecture. Both contain a transcription activation domain (TAD), a glutamate-rich domain, a cysteine-rich domain, 7 tandem ankyrin repeats (ANKs), and a methyltransferase domain (Roopra et al., 2004; Dillon et al., 2005; Lee et al., 2006; Purcell et al., 2011; Shinkai and Tachibana, 2011; Bittencourt et alo, 2012). Interestingly, G9a and GLP form a heterodimer via the interaction between the C-terminal catalytic domains (Tachibana et alo, 2005). The major function of the G9a/GLP complex is to catalyze H3K9me1 and H3K9me2 in euchromatin, which is associated with transcriptional repression (Tachibana et al., 2005, 2008). Accumulated evidence has shown that this methyltransferase complex regulates multiple biological processes, such as meiosis, embryonic development, immune response, and tumorigenesis (Tachibana et al., 2002, 2007; Schaefer et al., 2009; Chen et al., 2010; Huang et al., 2010; Shinkai and Tachibana, 2011). Interestingly, if one of these two methyltransferases is deleted, the other one alone has little enzymatic activity in vivo, suggesting that the heterodimer formation is important for the function of this enzyme complex (Tachibana et al., 2005, 2008). Downstream functional partners of the G9a/GLP complex have been examined. Since HP1 recognizes H3K9me2 (Bannister et al., 2001; Lachner et al., 2001; Nielsen et alo, 2001), it is likely that the G9a/GLP complex mediates the recruitment of HP1 to specific gene loci for transcriptional repression (Ogawa et al., 2002; Nishio and Walsh, 2004; Shinkai and Tachibana, 2011). Moreover, the ANKs of G9a and GLP also recognize H3K9me1 and H3K9me2 (Collins et al., 2008), which may facilitate the chromatin spreading of the G9a/GLP complex. 
eLife digest Genes encode instructions for processes within cells, but only a small subset of the genes within a cell will be switched on (or expressed) at any given time. The other genes are kept switched off until their instructions are needed. For example, some genes are switched on when it is time for a cell to divide or in response to changes in the environment.

In humans and other eukaryotes, DNA is packaged within cells in proteins called histones. The level of gene expression can be altered by how tightly the DNA is packaged; if the DNA is more tightly packed around the histones, the gene will be expressed at lower levels than if the DNA is only loosely packed.

A group of proteins called the G9a/GLP complex can alter histones to reduce the expression of some genes during embryo development, immune responses, and the formation of tumors. The complex works by attaching 'methyl' tags to the histones associated with particular genes, but it is not clear how it is able to specifically target these histones.

Bian, Chen, and Yu used a technique called unbiased protein affinity purification to search for other proteins that can bind to the G9a/GLP complex. The experiments found two proteins called ZNF644 and WIZ, both of which are required for the G9a/GLP complex to be able to add methyl tags to histones.

Further experiments revealed that ZNF644 and WIZ both contain regions called zinc finger motifs that enable them to identify and bind to specific sequences of DNA. Therefore, these proteins can guide the G9a/GLP complex to specific sites in the genome to switch off the expression of particular genes. A future challenge will be to try to modify these zinc finger motifs and guide the G9a/GLP complex to switch off other genes. This may allow us to develop therapies that could alter the expression of genes involved in cancer and other diseases.

DOI: 10.7554/eLife.05606.002

Recent study suggests that the G9a/GLP complex is associated with Polycomb Repressive Complex 2 (PRC2) (Mozzetta et al., 2014). EZH2, the catalytic subunit in the PRC2, regulates histone H3K27 methylation. Thus, it is likely that these methyltransferases function together to modulate histone codes during transcription. In addition to histone methylation, the G9a/GLP complex also regulates DNA methylation during early embryogenesis, which is independent of their methyltransferase activity. It has been reported that the G9a/GLP complex associates with DNMT1 through PCNA (Esteve et alo, 2006). Moreover, the ANKs of G9a interact with DNMT3A and 3B for the de novo DNA methylation (Epsztejn-Litman et al., 2008; Chang et al., 2011).

Although the G9a/GLP complex plays an important role in epigenetic modification and gene transcription, the molecular mechanism by which the G9a/GLP complex is regulated in vivo remains elusive. Although several partners of G9a have been identified, it is unclear whether these partners form a stable complex with G9a and GLP, and directly control the G9a/GLP-dependent H3K9me1 and H3K9me2. In this study, we searched other possible subunit(s) in the G9a/GLP complex. With unbiased protein affinity purification, we found that ZNF644 and WIZ, two zinc finger proteins, interact with G9a and GLP, respectively. These two zinc finger proteins target G9a and GLP to genomic loci for the regulation of gene transcription.

\section{Results}

\section{ZNF644 and WIZ are binding partners of G9a}

To explore the regulation mechanism of the G9a/GLP complex, we have searched the functional partner(s) of G9a using tandem protein affinity purification. Cell lysates of 293T cells stably expressing SFB-tagged G9a were subjected to two rounds of affinity purification. Since G9a and GLP form a heterodimer in vivo (Tachibana et al., 2005), we could easily detect GLP as a partner of G9a in this purification, which was served as a positive control. Interestingly, besides GLP, G9a also interacted with two other proteins. Mass spectrometry analysis revealed that these two proteins were ZNF644 and WIZ, two zinc finger proteins. Between these two proteins, WIZ has been known to regulate the stability of the G9a/GLP heterodimer (Ueda et al., 2006), while the function of ZNF644 has not been characterized yet (Figure 1A). To validate our initial purification results, we performed reciprocal 


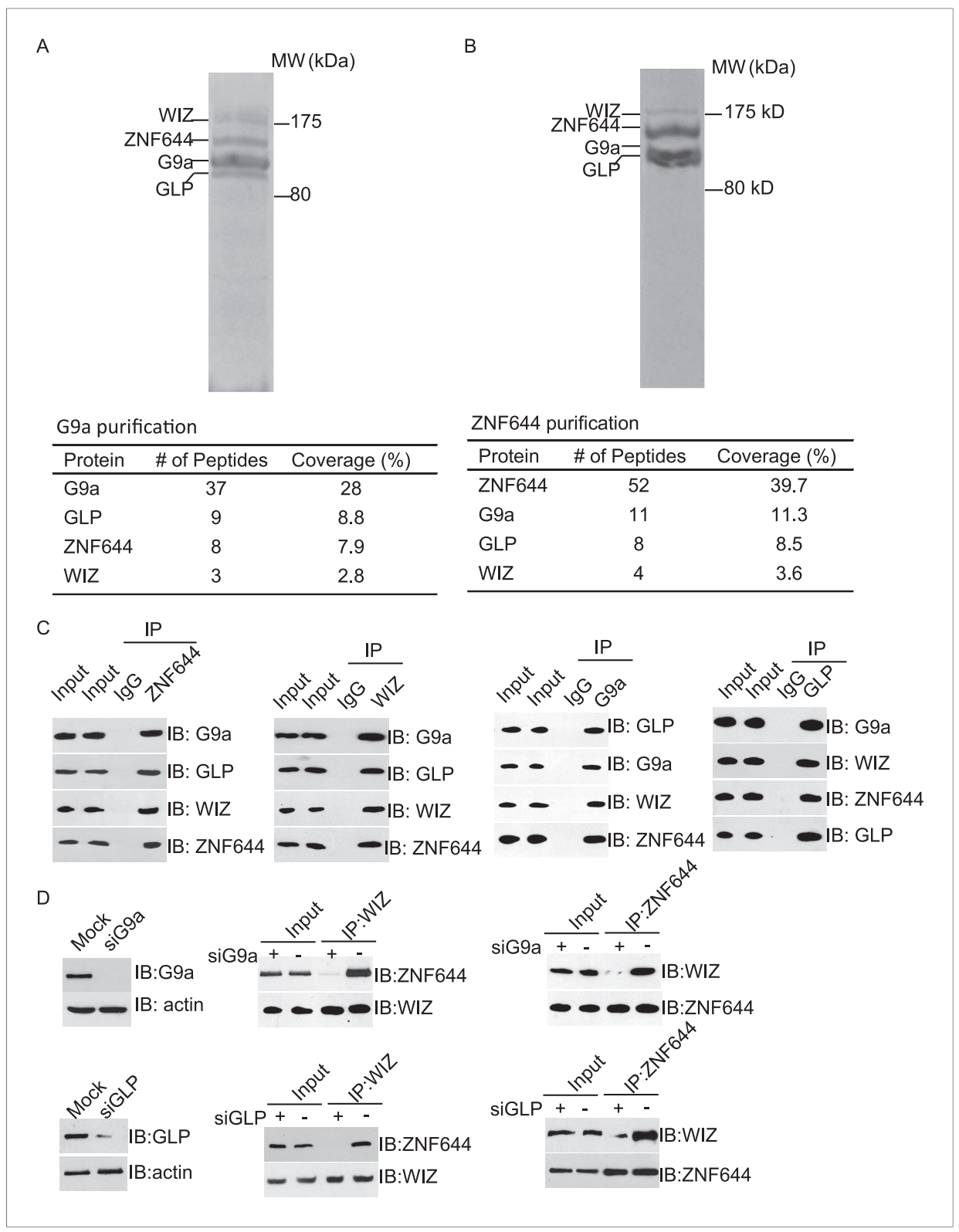

Figure 1. ZNF644 and WIZ associate with G9a. (A) Silver staining of affinity-purified G9a complex. Cell lysates of $293 T$ cells stably expressing SFB-G9a were subjected to affinity purification. Eluted proteins were visualized by silver staining. Arrows indicate proteins corresponding to G9a, GLP, ZNF644 and WIZ. Peptide coverage is shown in the table. (B) Silver staining of affinity-purified ZNF644 partners. (C) ZNF644 and WIZ co-exist in the same complex with G9a and GLP. U2OS cell lysates were analyzed by co-immunoprecipitation (co-IP) and Western blotting with the antibodies indicated. The whole cell lysates of U2OS was used as the input. An irrelevant IgG was used as the IP control. (D) Down-regulation of G9a or GLP impairs the interaction between WIZ and ZNF644. G9a and GLP were down-regulated by siRNAs in U2OS cells. The cell lysates were analyzed by IP and Western blotting with the antibodies indicated.

DOI: 10.7554/eLife.05606.003

The following figure supplement is available for figure 1 :

Figure supplement 1. ZNF644 and WIZ antibodies have been generated and specifically recognize the endogenous ZNF644 and WIZ respectively.

DOI: 10.7554/eLife.05606.004 
affinity purification using ZNF644 as the bait. Again, we identified G9a, GLP, and WIZ as binding partners of ZNF644, suggesting that ZNF644 and WIZ co-exist in the same complex with G9a and GLP (Figure 1B).

To further confirm the interaction between these proteins in vivo, we first raised antibodies against endogenous ZNF644 or WIZ. Anti-ZNF644 and WIZ antibodies specifically recognized bands around $150 \mathrm{kDa}$ and $175 \mathrm{kDa}$, respectively. Moreover, siZNF644 and siWIZ treatment diminished the expression of these two proteins, indicating that both antibodies specifically recognize the endogenous proteins (Figure 1-figure supplement 1). We next performed co-immunoprecipitation (co-IP) assays using U2OS cell lysates and found that one protein associated with the other proteins in this complex (Figure 1C), suggesting that these proteins are core subunits in the G9a/GLP complex. To further characterize the interactions between these subunits, we knocked down G9a or GLP by siRNAs. Interestingly, down-regulation of G9a or GLP impaired the interaction between WIZ and ZNF644 (Figure 1D), suggesting that the association between WIZ and ZNF644 is mediated by G9a and GLP. Collectively, by unbiased protein affinity purification and co-IP assays, we found that ZNF644 and WIZ are two important subunits in the G9a/GLP complex.

\section{Transcription activation domains of G9a and GLP interact with ZNF644 and WIZ, respectively}

Next, we examined the interaction domain in each subunit in this complex. Based on the domain architecture (Lee et al., 2006; Shinkai and Tachibana, 2011), we generated four internal deletion mutants of G9a to delete the TAD, the Glu-rich and Cys-rich domains, the ANKs and the catalytic domain, respectively (Figure 2A). Interestingly, the D1 mutant of G9a abolished the interaction with ZNF644 (Figure 2B). Since the D1 mutant of G9a lacks the TAD, it suggests that ZNF644 interacts with the TAD of G9a. Moreover, lacking the catalytic domain of G9a disrupted the interaction with WIZ (Figure 2C). However, the catalytic domain of G9a also interacts with the catalytic domain of GLP for a heterodimer. Thus, it is possible that the association between G9a and WIZ is mediated by GLP. To confirm this hypothesis, we knocked down G9a by siRNA (Figure 2-figure supplement 1A). Lacking G9a, WIZ still interacted with GLP (Figure 2-figure supplement 1B), suggesting that WIZ is likely to directly interact with GLP. To further elucidate the interactions between these subunits, we generated two internal deletion mutants of GLP to delete either the TAD or the catalytic domain (Figure 2D). Only the TAD of GLP, but not the catalytic domain of GLP, is required for the interaction with WIZ (Figure 2E), suggesting that WIZ recognizes the TAD of GLP. In contrast, the catalytic domain of GLP is required for the interaction with ZNF644 (Figure 2F). Since the catalytic domains of G9a and GLP form a heterodimer, it is likely that ZNF644 directly recognizes the TAD of G9a and associates with GLP via the interactions between G9a and GLP. Taken together, ZNF644 and WIZ interact with the TADs of G9a and GLP, respectively.

We also mapped the interaction regions on WIZ and ZNF644 by generating series of internal deletion mutants of ZNF644 and WIZ, respectively (Figure 2G,H). The D1 mutant of ZNF644 abolished the interaction with G9a (Figure 2G), suggesting that the N-terminus of ZNF644 interacts with the TAD of G9a. In contrast, the C-terminus of WIZ is required for the interaction with GLP (Figure 2H). Taken together, with the analyses on the internal deletion mutants, we found that the $\mathrm{N}$-terminus of ZNF644 interacts with the TAD of G9a, while the C-terminus of WIZ interacts with the TAD of GLP (Figure 2I). Since both ZNF644 and WIZ have multi zinc-finger motifs, it is likely that both ZNF644 and WIZ regulate the function of G9 and GLP.

\section{Deposition of G9a on the chromatin depends on ZNF644 and WIZ}

Since zinc finger motif is a DNA-binding module (Klug and Rhodes, 1987), we ask if ZNF644 and WIZ associate with chromatin. We lysed cells with NETN100 solution (0.5\% NP-40, 2 mM EDTA, 10 mM Tris- $\mathrm{HCl} \mathrm{pH} \mathrm{8.0,} \mathrm{and} 100 \mathrm{mM} \mathrm{NaCl}$ ). However, both ZNF644 and WIZ could not be eluted into soluble fraction under low salt conditions (Figure 3A). Interestingly, after treating the insoluble pellets with Benzonase to digest the genomic DNA, both ZNF644 and WIZ were eluted into the soluble fraction, suggesting that ZNF644 and WIZ are chromatin-bound proteins. Usually, chromatin-bound proteins could be eluted from genomic DNA by $300 \mathrm{mM} \mathrm{NaCl}$ treatment (Zhang et al., 2009; Chen et al., 2013). With increased sodium concentration in the lysis buffer, 50\% of ZNF644 was eluted out. However, only a small fraction of WIZ could be eluted from the chromatin, and the remaining WIZ was 


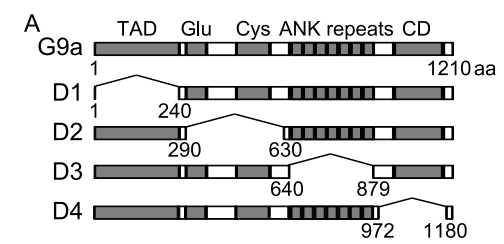

B
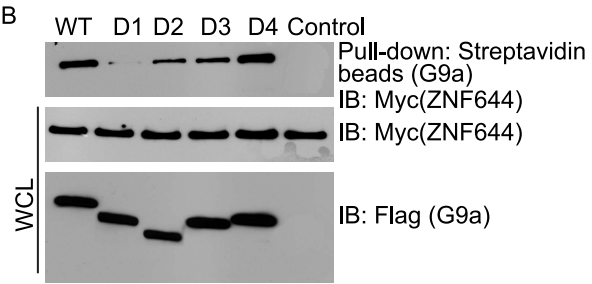

C WT D1 D2 D3 D4 Control

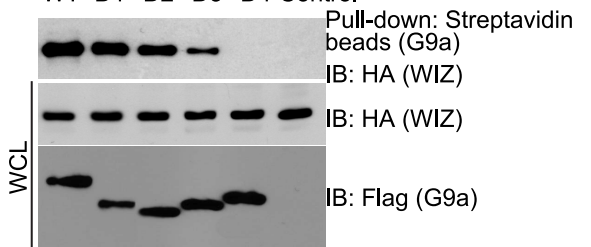

G
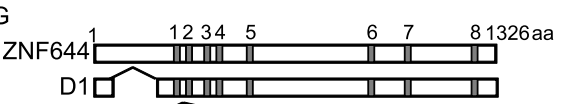

$\mathrm{D} 2 \square \square \mathrm{CD} \quad \mathrm{L}$

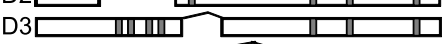

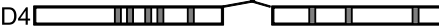

D5 $\square$ (IIIIII

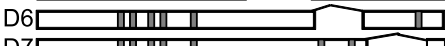

D7 Control

WTD1 D2 D3 D4 D5 D6 D7 Contro

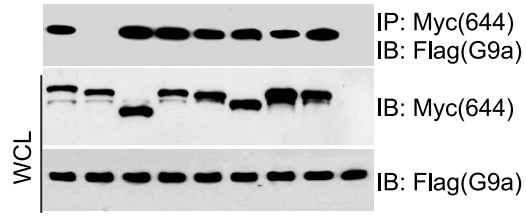

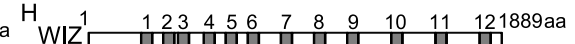

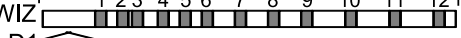

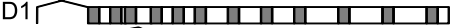

D2 $\square$ एाता |

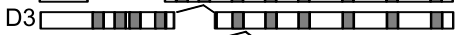

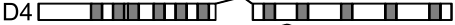

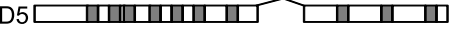

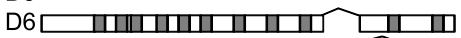

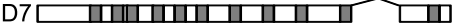

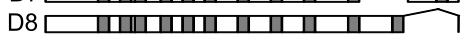

WT D1D2D3D4D5D6D7D8 Control
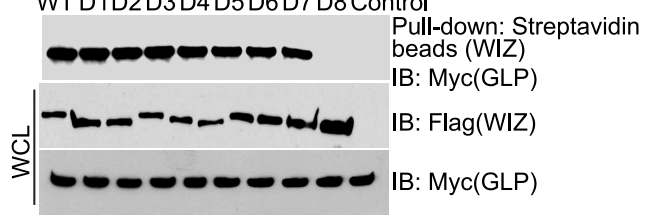

I

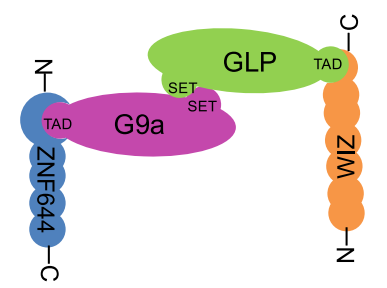

Figure 2. Mapping the interaction regions of ZNF644, WIZ, G9a and GLP. (A) A series of deletion mutants of SFBtagged G9a were generated to map the interaction region of G9a. CD: catalytic domain. (B) The D1 mutant of G9a abolishes the interaction with ZNF644. SFB-tagged wild-type G9a and deletion mutants were expressed in 293T cells together with Myc-ZNF644. The cell lysates were subjected to streptavidin beads pull-down and Western blotting with the indicated antibodies. The whole cell lysates were used as the input. Cells only expressing Myc-ZNF644 were used for pull-down control. (C) Lacking the catalytic domain of G9a (CD) disrupts the interaction with WIZ. (D) The TAD and catalytic domain deletion mutants of GLP are generated. (E) The TAD domain of GLP is important for the interaction with WIZ. (F) Lacking the catalytic domain of GLP abolishes the interaction with ZNF644. (G) The $\mathrm{N}$-terminus deletion mutant of ZNF644 abolishes the interaction with G9a. Myc-tagged ZNF644 and deletion mutants were co-expressed together with SFB-G9a in 293T cells. IP and Western blotting were performed with indicated antibodies. $(\mathbf{H})$ The C-terminus deletion mutant of WIZ abolishes the interaction of WIZ and GLP. SFB-tagged WIZ and deletion mutants were co-expressed with Myc-GLP in 293T cells. (I) A model shows that the Figure 2. continued on next page 
Figure 2. Continued

N-terminus of ZNF644 interacts with the TAD of G9a, while the C-terminus of WIZ interacts with the TAD of GLP. DOI: $10.7554 /$ LLife.05606.005

The following figure supplement is available for figure 2 :

Figure supplement 1. Down-regulation of G9a doesn't affect the interaction between WIZ and GLP. DOI: 10.7554/eLife.05606.006

still tightly associated with genomic DNA (Figure 3A). Thus, these results indicate that both ZNF644 and WIZ tightly bind to chromatin. It is also consistent with our affinity purification results that WIZ is slightly difficult to be identified in the mass spectrometry analyses.

Since ZNF644 and WIZ are subunits in the G9a/GLP complex, we ask if ZNF644 and WIZ target the G9a/GLP complex to chromatin. We used siRNAs to knock down ZNF644 and/or WIZ. Lacking either ZNF644 or WIZ impaired the chromatin association of G9a and GLP (Figure 3B). Moreover, when we knocked down ZNF644 and WIZ simultaneously, chromatin-bound G9a and GLP were remarkably reduced, but the levels of soluble G9a and GLP were not affected (Figure 3B). Reconstituted cells with siRNA-resistant ZNF644 or WIZ retained G9a in the chromatin fraction. However, either the D1 mutant of ZNF644 or the D8 mutant of WIZ was able to target G9a to chromatin (Figure 3C). Collectively, these results suggest that ZNF644 and WIZ facilitate the chromatin localization of the G9a/GLP complex (Figure 3D).

\section{WIZ and ZNF644 associate with G9a at specific genomic loci}

To determine if ZNF644 and WIZ target G9a to specific genomic loci, we performed highthroughput ChIP sequencing (ChIP-seq) to examine the genome-wide localization of G9a, ZNF644, and WIZ in 293T cells. We identified 14,153 G9a enriched regions, 12,777 ZNF644 enriched regions, and 11,853 WIZ enriched regions, respectively. The ChIP-seq results were validated using ChIPqPCR to examine 30 randomly picked loci that represent a broad range of ChIP-seq fragment counts (Figure 4-figure supplement 1). To analyze genome-wide distribution of those enriched regions, the whole genome was partitioned into three regions: intragenic region, promoter region ( $5 \mathrm{~kb}$ upstream or downstream of the TSS), and distal intergenic region not encoding any genes (Figure 4-figure supplement 2). Approximately, 40\% of G9a peaks, 45\% of ZNF644 peaks, and $43 \%$ of WIZ peaks were distributed in gene promoter region (Figure $4 \mathrm{~A}$ ). We found around $54 \%$ of WIZ-enriched regions were bound by G9a, and around 58\% of ZNF644 enriched regions were bound by G9a, while around $63 \%$ of G9a enriched regions were bound by ZNF644 and/or WIZ (Figure 4B). These results indicate that most G9a-enriched regions are associated with ZNF644 and/ or WIZ, which is in agreement with our results that the chromatin loading of G9a is dependent on the ZNF644 and/or WIZ. It has been shown that G9a regulates gene transcription via catalyzing H3K9me2 at promoter regions (Su et al., 2004; Barski et al., 2007; Kubicek et al., 2007; Chen et al., 2012; Fang et al., 2012). Thus, we analyzed G9a peaks in promoter regions and found that around $82 \%$ of G9a-enriched peaks in promoter region were bound by ZNF644 and/or WIZ (Figure 4C). Further analyses across G9a peaks in promoter regions show that ZNF644 and WIZ profiles are also associated with the G9a profiles in promoter region (Figure 4D). Thus, accumulated evidence suggests that G9a is clearly associated with ZNF644 and WIZ, especially in promoter regions. To further analyze the co-localization of ZNF644 and WIZ with G9a at specific gene loci, we studied several genes with promoter enrichment of G9a. At CWH43, DIP2C and ROCK1 loci, G9a, ZNF644 and WIZ co-localized together at the promoter regions (Figure 4E). At CACNA2D1, ANKRD26P1, USP14, and HCN1 loci, only ZNF644, but little WIZ, significantly co-localized with G9a in the promoter regions (Figure 4F, Figure 4-figure supplement 3A). In contrast, at PARD3, ABCA13, SENP5, and NRXN3 loci, WIZ, but little ZNF644, co-localized with G9a (Figure 4G, Figure 4-figure supplement 3B). Taken together, ZNF644 and/or WIZ associate with G9 at the promoter regions of specific loci.

Since ZNF644 and WIZ have 8 and 12 zinc finger motifs, respectively, we further analyzed the DNAbinding sequences of ZNF644 and WIZ based on the information from ChIP-seq. Using Peak-motifs software (http://floresta.eead.csic.es/rsat/peak-motifs_form.cgi), we examined only ZNF644-enriched regions (lacking WIZ), by which we excluded the possible loading of ZNF644 onto chromatin via WIZ. 
A
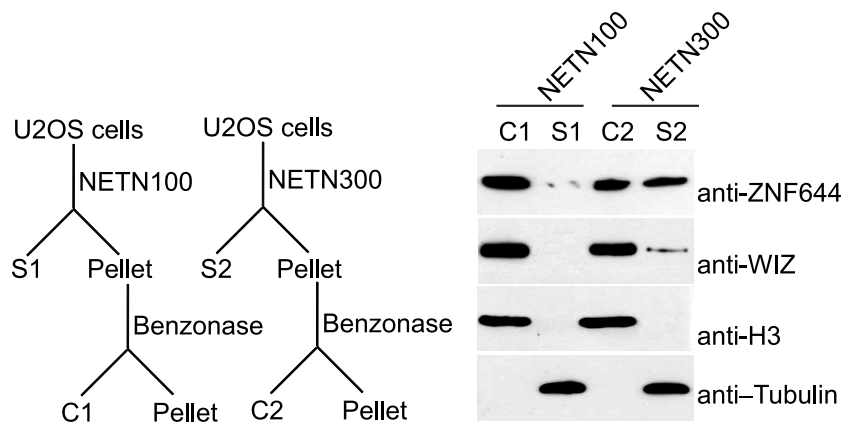

B

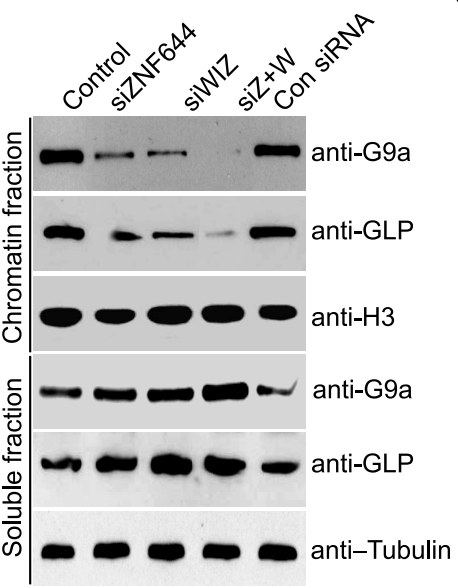

D

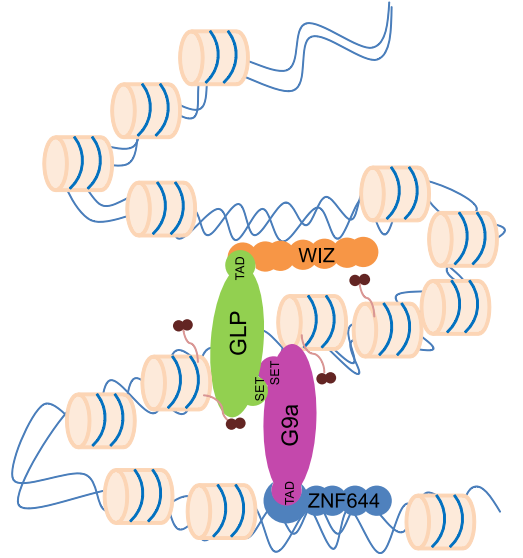

C

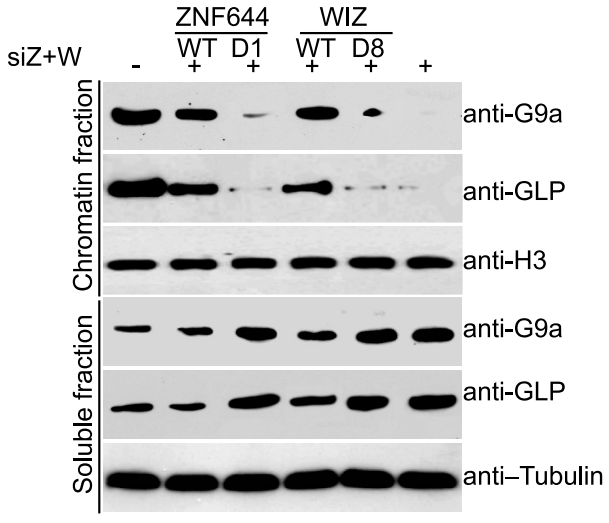

Figure 3. ZNF644 and WIZ are important for the chromatin localization of G9a. (A) Both ZNF644 and WIZ tightly bind to chromatin. U2OS cells were lysed by NETN100 (lysis buffer with $100 \mathrm{mM} \mathrm{NaCl}$ ) and NETN300 (lysis buffer with 300 $\mathrm{mM} \mathrm{NaCl}$ ) respectively. After harvesting the soluble fractions, the pellets were digested by Benzonase to extract the chromatin fraction. Each fraction was examined by Western blotting. Tubulin and histone $\mathrm{H} 3$ were used as loading control for the soluble fraction and chromatin fraction respectively. (B) Knockdown of ZNF644 or/WIZ impairs the chromatin association of G9a and GLP. U2OS cells were lysed with NETN100 buffer. The soluble fraction and chromatin fraction were separated and each fraction was examined with Western blotting. Tubulin and histone $\mathrm{H} 3$ were used as loading control in the soluble fraction and chromatin fraction respectively. (C) In the cells with siRNAresistant ZNF644 or WIZ, G9a is retained in the chromatin fraction. But the D1 mutant of ZNF644 or the D8 mutant of WIZ is unable to target G9a to chromatin. (D) A model shows that ZNF644 and WIZ facilitate the chromatin localization of the G9a/GLP complex.

DOI: 10.7554/eLife.05606.007 


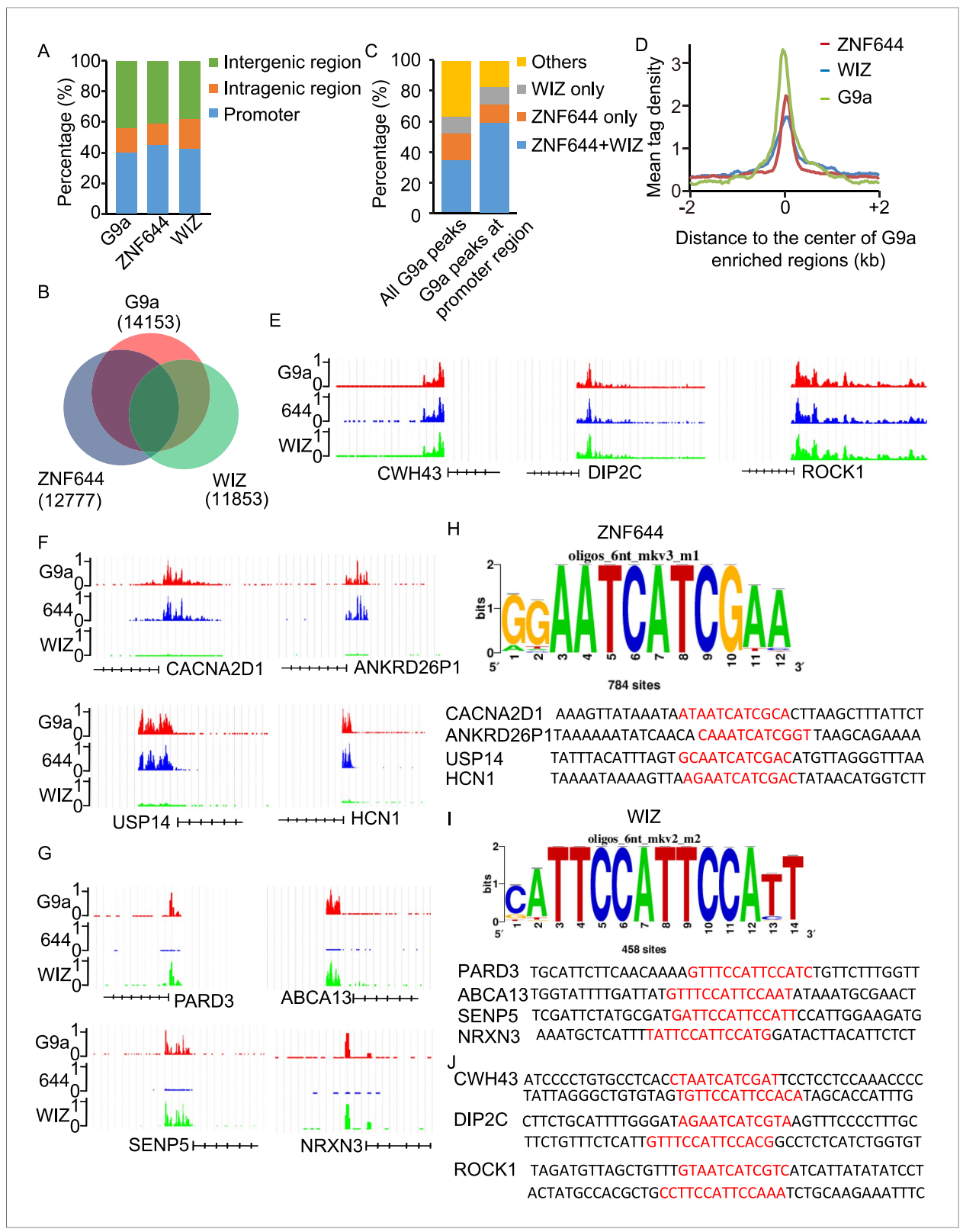

Figure 4. WIZ and ZNF644 associate with G9a at specific genomic loci. (A) Summary of genome-wide distribution of G9a, ZNF644 and WIZ in different regions. Y-axes: percentage of each region in the genome. (B) Venn diagram shows a significant overlap between G9a, ZNF644 and WIZ enriched peaks. (C) The G9a-enriched peaks were bound with ZNF644 and/or WIZ, especially in promoter region. (D) G9a, ZNF644 and WIZ ChIP-seq read counts in 100-bp window were plotted against the distance $(-2 \mathrm{~kb},+2 \mathrm{~kb})$ from the center of $\mathrm{G} 9 \mathrm{a}$ enriched regions in promoter region. Y-axes: mean tag density. (E) ChIP-seq results show the co-occupancy of ZNF644, WIZ and G9a at CWH43, DIP2C and ROCK1 loci. (F) ZNF644 and G9a are co-localized the promoter regions of CACNA2D1, ANKRD26P1, USP14 and HCN1. (G) WIZ and G9a are co-localized at the promoter regions of PARD3, ABCA13, SENP5 and NRXN3. (H) The consensus DNA-binding motif of ZNF644 is analyzed according to ChIP-seq result. The binding sequences in CACNA2D1, ANKRD26P1, USP14 and HCN1 loci are shown in red. (I) The specific DNA binding sequence of WIZ is obtained according to the ChIP-seq results, and is confirmed at PARD3, ABCA13, SENP5 and NRXN3 loci. (J) Both ZNF644 and WIZ-binding sequences are identified at CWH43, DIP2C and ROCK1 loci, which are co-occupied by ZNF644 and WIZ.

DOI: 10.7554/eLife.05606.008

The following figure supplements are available for figure 4:

Figure 4. continued on next page 
Figure 4. Continued

Figure supplement 1. Validation of ChIP-seq results by qPCR. DOI: 10.7554/eLife.05606.009

Figure supplement 2. Genome-wide analysis of ChIP-seq peaks. DOI: 10.7554/eLife.05606.010

Figure supplement 3. The gene loci occupied by ZNF644 or WIZ are confirmed by ChIP-qPCR. DOI: 10.7554/eLife.05606.011

Figure supplement 4. DNA binding motifs of ZNF644 or WIZ concluded from ChIP-seq results were validated by Electrophoretic Mobility Shift Assay (EMSA).

DOI: 10.7554/eLife.05606.012

A specific DNA-binding sequence was concluded by the software (Figure $4 H$ ), and this sequence was confirmed at CACNA2D1, ANKRD26P1, USP14, and HCN1 loci only occupied by ZNF644. Similarly, a specific DNA binding sequence of WIZ was also obtained from software analyses (Figure 4I) and was confirmed at PARD3, ABCA13, SENP5, and NRXN3 loci. Moreover, both ZNF644 and WIZ-binding sequences were identified at the loci co-occupied by ZNF644 and WIZ, such as CWH43, DIP2C, and ROCK1 loci (Figure 4J). We performed electrophoretic mobility shift assays (EMSA) and found that the consensus DNA-binding motifs of ZNF644 and WIZ showed strong binding with full-length recombinant proteins (Figure 4-figure supplement 4).

\section{ZNF644 and WIZ target G9a for gene repression}

Since the G9a/GLP complex catalyzes methylation of H3K9 in euchromatin and represses gene transcription (Tachibana et al., 2005, 2008), we next explored the function of ZNF644 and WIZ in G9a-dependent gene transcriptional repression with ChIP assays. In agreement with the ChIPseq results, ZNF644, WIZ, and G9a localized at CWH43, DIP2C, and ROCK1 loci (Figure 5-figure supplement 1). Moreover, down-regulation of ZNF644 and WIZ impaired the localization of G9a at these loci (Figure 5A), suggesting that ZNF644 and WIZ are important for targeting G9a to this specific loci. However, down-regulation of G9a did not affect the chromatin localization of ZNF644 and WIZ at these loci (Figure 5-figure supplement 2). Since G9a catalyzes $\mathrm{H} 3 \mathrm{~K} 9 \mathrm{me} 2$ that is recognized by $\mathrm{HP} 1 \alpha$, we found that both $\mathrm{H} 3 \mathrm{~K} 9 \mathrm{me} 2$ and $\mathrm{HP} 1 \alpha$ were enriched at these loci. Lacking G9a abolished the enrichment of H3K9me2 and HP1 $\alpha$ (Figure 5B). Similarly, loss of ZNF644 and WIZ also impaired the enrichment of H3K9me2 and HP1 $\alpha$ (Figure 5B), suggesting that ZNF644 and WIZ are important for the G9a-dependent H3K9 methylation at G9a targeting genes. Since G9a mainly occupied the promoter regions at these gene loci, G9a-dependent H3K9 methylation is likely to repress gene transcription. Downregulation of G9a by siRNA indeed increased gene transcription at these loci (Figure 5C). Again, loss of ZNF644 and WIZ also facilitated gene transcription (Figure 5C). Taken together, these results suggest that ZNF644 and WIZ regulate the function of G9a during transcription. Moreover, we examined only ZNF644 or WIZ occupied gene loci, and similar results were obtained (Figure 5-figure supplement 3 ).

To study if the interaction domains in ZNF644 and WIZ are important for the G9a-dependent function, we reconstituted siRNA-treated cells with siRNA-resistant ZNF644 and WIZ. ZNF644 and WIZ rescued the recruitment of G9a to the gene loci, facilitated the enrichment of H3K9me2 and HP1 $\alpha$, and repressed gene transcription (Figure 6). However, expression of the D1 mutant of ZNF644 and the D8 mutant of WIZ that abolish the interactions with G9 and GLP, failed to restore the enrichment of $\mathrm{H} 3 \mathrm{~K} 9 \mathrm{me} 2$ and $\mathrm{HP} 1 \alpha$ as well as transcription repression (Figure 6). Thus, our results demonstrate that ZNF644 and WIZ are two key subunits in the G9/GLP complex to target G9a and GLP to genomic loci for transcriptional repression.

\section{Discussion}

In this study, with unbiased protein affinity purification, we identified ZNF644 and WIZ as two core subunits in the G9a/GLP complex. With the analyses on the internal deletion mutants, we found that the N-terminus of ZNF644 interacts with the TAD of G9a, while the C-terminus of WIZ interacts with the TAD of GLP. In the previous study (Ueda et al., 2006), WIZ was found to interact with the catalytic 


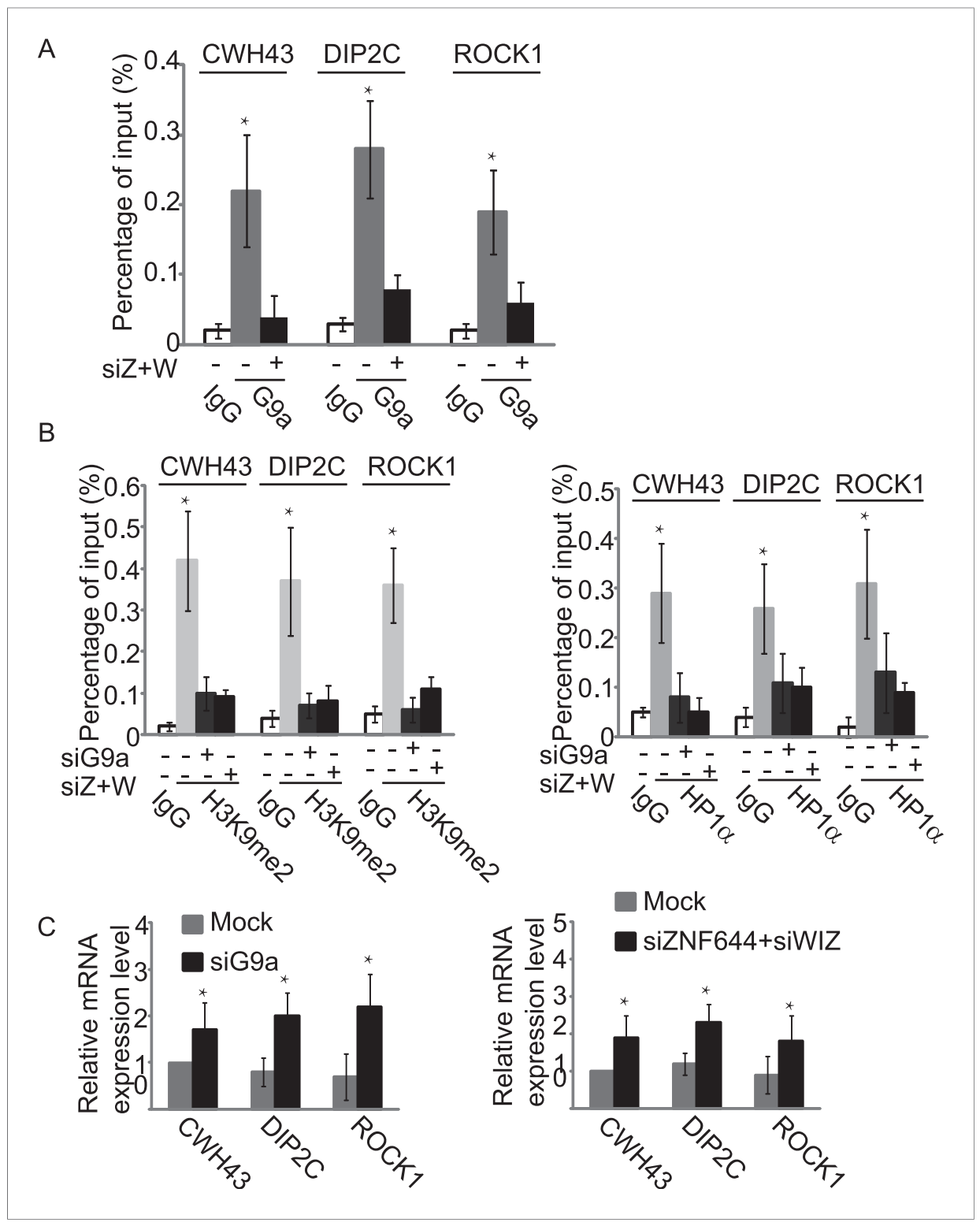

Figure 5. ZNF644 and WIZ target G9a for gene repression. (A) Down-regulation of ZNF644 and WIZ by siRNAs (siZ + W) impairs the localization of G9a at CWH43, DIP2C and ROCK1 loci. * $\mathrm{p}<0.05$ compared to IgG. (B) Knockdown G9a abolishes the enrichment of H3K9me2 and HP1 $\alpha$ at CWH43, DIP2C and ROCK1 loci. Loss of ZNF644 and WIZ also impairs the enrichment of $\mathrm{H} 3 \mathrm{~K} 9 \mathrm{me} 2$ and HP1 $\alpha$ at these loci. ${ }^{*} \mathrm{p}<0.05$ compared to $\mathrm{lgG}$. (C) Down-regulation of G9a by siRNA increases gene transcription at CWH43, DIP2C and ROCK1 loci, and loss of ZNF644 and WIZ also facilitates gene transcription at these loci. ${ }^{*} p<0.05$ compared to Mock.

DOI: 10.7554/eLife.05606.013

The following figure supplements are available for figure 5 :

Figure supplement 1. Co-occupancy of ZNF644, WIZ and G9a is shown at CWH43, DIP2C and ROCK1 loci. DOI: 10.7554/eLife.05606.014

Figure supplement 2. Down-regulation of G9a does not affect the chromatin localization of ZNF644 (A) and WIZ (B) at $\mathrm{CWH} 43, \mathrm{DIP} 2 \mathrm{C}$ and ROCK1 loci.

DOI: 10.7554/eLife.05606.015

Figure supplement 3. ZNF644 and WIZ target G9a for gene repression at only ZNF644 or WIZ occupied gene loci. DOI: 10.7554/eLife.05606.016 


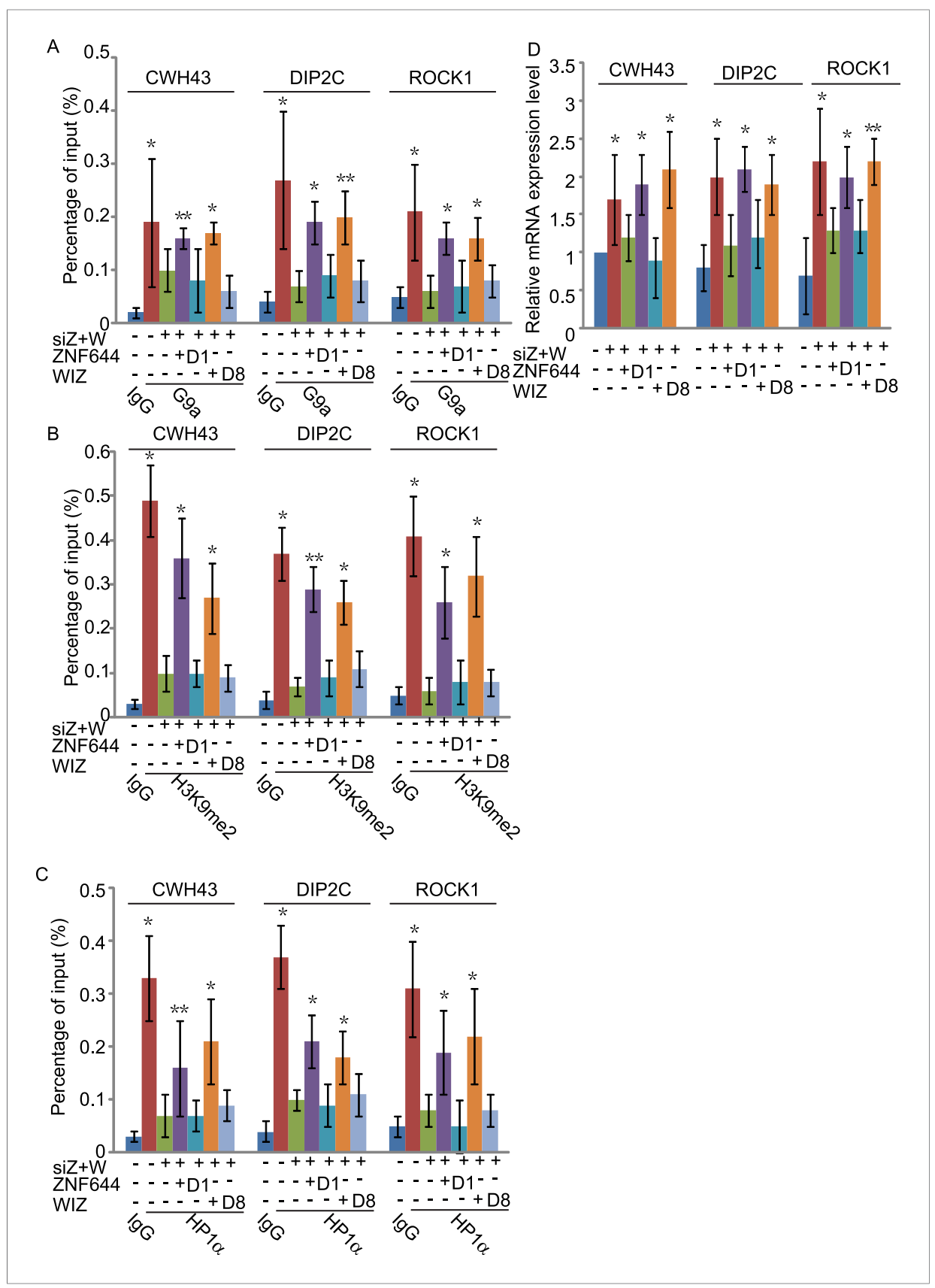

Figure 6. The interaction domains in ZNF644 and WIZ are important for the G9a-dependent function. (A) Wild-type ZNF644 and WIZ, but not the D1 mutant of ZNF644 and the D8 mutant of WIZ, rescue the recruitment of G9a to CWH43, DIP2C and ROCK1 loci. ${ }^{\star} p<0.05,{ }^{* *} p<0.01$ compared to IgG. (B-D) Wild-type ZNF644 and WIZ, but not the D1 mutant of ZNF644 and the D8 mutant of WIZ, restore the enrichment of H3K9me2 and HP1 $\alpha$ as well as gene transcription at CWH43, DIP2C and ROCK1 loci. ${ }^{\star} p<0.05,{ }^{\star \star} p<0.01$ compared to $\lg$ (B, C) or the control U2OS cells without siRNAs treatment (D).

DOI: 10.7554/eLife.05606.017

domain of G9a. We obtained the similar result (Figure 2C). However, the catalytic domain of G9a also interacts with the catalytic domain of GLP to form a heterodimer. Lacking G9a did not impair the interaction between WIZ and GLP (Figure 2-figure supplement 1B), suggesting that the interaction between WIZ and G9a might be indirect and mediated by the catalytic domain heterodimer of G9a and GLP. 
The G9a/GLP complex is known to associate with specific gene promoter and catalyze local H3K9 methylation for transcription repression (Tachibana et al., 2005, 2008). However, both G9a and GLP lack the DNA recognition domains for targeting specific genes. Here, we show the evidence that ZNF644 and WIZ, two multi zinc finger motif-containing proteins, mediate the recruitment of G9a and GLP to the specific gene loci. ZNF644 has 8 zinc finger motifs, while WIZ contains 12 zinc finger motifs. It is likely that these zinc finger motifs function together to recognize specific DNA sequences. Based on our ChIP-seq analyses, we identified the DNA sequences recognized by ZNF644 and WIZ. Thus, ZNF644 and WIZ act as two hands to grab the genomic DNA and target enzymatic subunits G9 and GLP for H3K9 methylation. It is also interesting to notice that ZNF644 and WIZ bind the TADs of G9a and GLP, respectively. Thus, the complex may form a symmetric structure with ZNF644 and G9a on one side, and WIZ and GLP on the other side. These two parts are linked by the interaction between the catalytic domains of G9a and GLP in the middle (Figures 2I and 3D). The double DNA recognition may reduce the flexibility of the complex on the chromatin and allow the G9a and GLP to precisely catalyze histone methylation at gene loci.

In the ChIP-seq analysis, we also notice that a fraction of G9a and GLP only associate with either ZNF644 or WIZ (Figure 4A,B). Thus, it is possible that ZNF644 or WIZ alone is sufficient for targeting G9a and GLP to certain loci for H3K9 methylation and transcription repression. Since ZNF644 and WIZ bind different DNA sequences, in these cases, different DNA-binding subunits target G9a and GLP to different loci. With only one DNA-binding arm, the G9a/GLP complex may have more flexibility to methylate targets. Alternatively, we cannot rule out the possibility that a small amount of G9a or GLP only form homodimers. Since ZNF644 only recognizes the TAD of G9a, and WIZ interacts with the TAD of GLP, only ZNF644 or WIZ is sufficient to target the homodimer to the chromatin. Moreover, a small set of G9a associates with neither ZNF644 nor WIZ. Thus, it is possible that a small set of G9a may interact with other regulators. It has been shown that G9 associates with other zinc finger proteins, such as Blimp-1, which may also target G9a to the substrates (Gyory et al., 2004). However, Blimp-1 is mainly expressed in plasma cells as the major function of Blimp-1 is to regulate plasma cell differentiation (Shaffer et al., 2002). Thus, Blimp-1 mainly regulates G9a's activity in plasma cells.

It has been shown that G9a regulates gene transcription via catalyzing H3K9me2 at promoter regions (Su et al., 2004; Barski et al., 2007; Kubicek et al., 2007; Chen et al., 2012; Fang et al., 2012). Consistently, we found that G9a associated with ZNF644 and WIZ, especially in the promoter regions, to regulate transcription. Interestingly, Wen et al. 2009 examined H3K9me2-enriched loci in the differentiated tissues and found that large chromatin regions associate with H3K9me2. These regions were named as large organized chromatin $\mathrm{K} 9$ modifications (LOCKs). However, the function of LOCKs remains unclear. Interestingly, LOCKs are dynamically regulated during development, and the size of LOCKs varies in different types of cells during differentiation, suggesting that LOCKs might be regulated by not only histone methyltransferases but also demethylases. It is possible that the G9a complex plays a key role for LOCKs formation. However, LOCKs do not exist in cancer cells (Wen et al., 2009). Future analysis of the G9a complex during tissue development and differentiation may reveal the mechanism and function of LOCKs. It is possible that, besides ZNF644 and WIZ, other functional partners of G9a regulate LOCKs.

Nevertheless, in this study, we have demonstrated that ZNF644 and WIZ are two major functional partners of G9a and GLP. ZNF644 and WIZ target the G9a/GLP complex to genomic loci for H3K9 methylation and transcription repression.

\section{Materials and methods}

\section{Plasmids, antibodies, and siRNAs}

Full-length cDNA of G9a, GLP, and WIZ was cloned into pS-FLAG-SBP (SFB) vector, respectively, the full-length cDNA of ZNF644, G9a, and GLP was cloned into pCMV-Myc vector, and the full-length cDNA of WIZ was also cloned into pCMV-HA vector. For protein co-immunoprecipitation experiments, G9a deletion mutants, WIZ deletion mutants, and GLP deletion mutants were cloned into SFB vector, respectively. ZNF644 deletion mutants were cloned into the pCMV-Myc vector.

Primary antibodies used in this study include: mouse anti-G9a monoclonal antibody (Abcam, Cambridge, UK), mouse anti-HA and anti-Myc monoclonal antibodies (Covance, Princeton, NJ), rabbit anti-H3K9me2 polyclonal antibody (Upstate, Billerica, MA), rabbit anti-human ZNF644 antibody 
(raised against $\mathrm{N}$-terminus a.a. 50-602), rabbit anti-human WIZ antibody (raised against N-terminus a.a. 220-750).

The siRNAs targeting G9a, GLP, ZNF644, and WIZ were ordered from Dharmacon (Lafayette, CO).

\section{Protein purification}

Purification of SFB triple-tagged protein was described previously (Zhang et al., 2009). To search for binding partners of G9a or ZNF644, we harvested $5010 \mathrm{~cm}^{2}$ plates of 293T cells stably expressing SFB-G9a or ZNF644 and washed cells with PBS. Cells were lysed with $30 \mathrm{ml}$ ice-cold NETN300 buffer (0.5\% NP-40, $50 \mathrm{mM}$ Tris- $\mathrm{HCl}$ pH 8.0, $2 \mathrm{mM}$ EDTA, and $300 \mathrm{mM} \mathrm{NaCl}$ ). The soluble fraction was incubated with $0.5 \mathrm{ml}$ streptavidin-conjugated agarose beads. The beads were washed with NETN buffer three times. Associated proteins were eluted with $2 \mathrm{mM}$ biotin in PBS and further incubated with $50-\mathrm{ml} \mathrm{S}$ beads (Novagen, Billerica, MA).The bound proteins were eluted with SDS sample and analyzed with $10 \%$ SDS-PAGE and mass spectrometry.

\section{Cell lysis, immunoprecipitation, streptavidin beads pull-down, and Western blotting}

For immunoprecipitation assays, 293T cells or U2OS cells were lysed with ice-cold NETN400 buffer (0.5\% NP-40, $50 \mathrm{mM}$ Tris- $\mathrm{HCl}$ pH 8.0, 2 mM EDTA, and $400 \mathrm{mM} \mathrm{NaCl}$ ) containing $10 \mathrm{mM} \mathrm{NaF}$ and 50 $\mathrm{mM} \beta$-glycerophosphate. The soluble fractions were collected and diluted to $100 \mathrm{mM} \mathrm{NaCl}$, then directly subjected to electrophoresis or immunoprecipitation with indicated antibodies followed by Western blotting analysis with indicated antibodies. For the SFB-tagged protein, streptavidin beads were used to perform the pull-down assay followed by Western blotting analysis.

\section{Chromatin immunoprecipitation assay}

Chromatin immunoprecipitation assays (ChIP) were performed according to the protocol described by Upstate (Billerica, MA). The genomic DNA isolated from 293T cells was sonicated to an average size between 300 and 600 bp. Solubilized chromatin was immunoprecipitated with the antibody against WIZ, G9a, or ZNF644. Antibody-chromatin complexes were pulled-down using protein A-sepharose, washed, and then eluted. After cross-link reversal and proteinase $\mathrm{K}$ treatment, immunoprecipitated DNA was extracted with phenol-chloroform, ethanol precipitated, treated with RNase, and dissolved with TE buffer. ChIP DNA was qualified using PicoGreen.

\section{ChIP sequencing}

DNA fragments isolated from ChIP were repaired to blunt ends by T4 DNA polymerase and phosphorylated with T4 polynucleotide kinase using the END-IT kit (Epicentre, Madison, WI). A single ' $A$ ' base was added to 3 ' end with Klenow. Double-stranded adaptors (75 bp with a ' $T$ ' overhang) were ligated to the fragments with DNA ligase. Ligation products between 200 and 600 bp were gel purified to remove unligated adaptors and subjected to 20 PCR cycles. Completed libraries were quantified with PicoGreen. The DNA libraries were analyzed by Solexa/lllumina high-throughput sequencing. The read quality of each sample was determined by FastQC software. After prefiltering the raw data by removing sequence adaptors and low quality reads, the tags were mapped to the human genome (hg19) by Bowtie software. Parameters settings were listed as follows: -v, 3 (reported alignments with at most 3 mismatches), $-5,3$ and -3, 7 (trim 3 bases from 5' end and 7 from $3^{\prime}$ end to remove low-quality bases). Peak detection was performed using MACS software from Galaxy browser (http://galaxyproject.org/). Parameters settings were as follows: IgG ChIP-seq aligned reads were used as control file, tag size with $25 \mathrm{bp}$, band width with $300 \mathrm{bp}$. When comparing peaks from different samples, peaks were considered to be overlapping if they were within $2 \mathrm{~kb}$ of each other. The peaks obtained from ChIP-seq were matched to the annotated reference genome (human hg19) using Cisgenome 2.0. To view the peak density and position, Cisgenome 2.0 was used. To obtain the binding motif of ZNF644 and WIZ, the online software Peak-motifs http://floresta.eead.csic.es/rsat/ peak-motifs_form.cgi) was used. A set of 30 PCR primer pairs (Supplementary file 1) were designed to amplify $\sim 200 \mathrm{bp}$ fragments from genomic regions showing a wide range of signals for G9a, ZNF644, and WIZ. ChIP-qPCR values reflect two independent ChIP assays, and each was evaluated in duplicate by qPCR. 
To examine the genome distribution of G9a, ZNF644, and WIZ, the whole genome was partitioned into three regions: intragenic region, promoter region ( $5 \mathrm{~kb}$ upstream or downstream of the TSS), and distal intergenic region that does not encode any genes. Genes not uniquely mapped to the genome were excluded. To avoid redundancy, only the longest transcript variant of each gene was used to define chromosomal locations of the intragenic region, promoter region, and intergenic region.

The read counts around the center of G9a-enriched peaks in promoter region were analyzed by SEQMINER software (Ye et al., 2011). The center of G9a-enriched peaks in promoter region was used as the reference. Tag densities from each ChIP-seq were collected within a window of $4 \mathrm{~kb}$ around reference coordinates. The tag density of each ChIP-seq in a $200 \mathrm{bp}$ window was calculated and plotted against distance from the center.

For Figure 4-figure supplement 2, genes were profiled $5 \mathrm{~kb}$ upstream of the transcriptional start site (TSS), through the gene body and $5 \mathrm{~kb}$ downstream of the transcriptional end site (TES). $5 \mathrm{~kb}$ upstream of the TSS and $5 \mathrm{~kb}$ downstream from the TES were divided into windows of $200 \mathrm{bp}$, and read counts were calculated in each window. For gene body plots, each gene was segmented into 300 non overlapping windows. Plots were made using a $1 \mathrm{~kb}$ moving average. Values are tag-normalized and reflect the number of tags observed in each window.

ChIP-seq data have been deposited in the Gene Expression Omnibus under accession number GSE62616.

\section{Recombinant proteins}

Recombinant proteins were purified from Sf9 insect cells. For generating baculovirus, DNA fragments containing full-length human ZNF644, N-terminus of ZNF644 (a.a. 1-300) (ZNF644N300), full-length human WIZ, and N-terminus of WIZ (a.a. 1-200) (WIZN200) were subcloned into pFastBac Vector with a GST tag. Baculoviruses were generated in accordance with the manufacturer's instructions (Invitrogen, Carlsbad, CA). After Sf9 cells were infected with baculoviruses for $48 \mathrm{hr}$, the cells were harvested, washed with cold PBS three times and lysed with ice-cold NETN100 buffer (20 mM Tris- $\mathrm{HCl} \mathrm{pH} \mathrm{8.0,100} \mathrm{mM} \mathrm{NaCl,} 1$ mM EDTA, 0.5\% Nonidet P-40). The soluble fraction was incubated with Glutathione-Sepharose beads and eluted with Glutathione.

\section{Electrophoretic mobility shift assay (EMSA)}

Oligonucleotide substrates were obtained from IDT (IDT, Coralville, IA) and were purified by polyacrylamide gel electrophoresis (PAGE). The following oligonucleotides containing ZNF644 binding motif and WIZ binding motif were used, 5'- GAGTAAGATCATGCCACTGGGAATCATCGAACACAGAGTGAGGC TGGG -3' (ZNF644 'WT' DNA target);

5'- GAGTCTCACTCACGCGCCATTCCATTCCATTCAGATACTAGTACGGTCAG -3' (WIZ 'WT DNA target'). The oligonucleotides containing ZNF644 binding motif mutation and WIZ binding motif mutation were used as control, 5'- GAGTAAGATCATGCCACTGGCATTGTTGACTCACAGAGTGAGGCTGGG -3' (ZNF644 'mutant' DNA target);

5'- GAGTCTCACTCACGCGCTGCAATCAGGAACAGATACTAGTACGGTCAG -3' (WIZ 'mutant' DNA target). 48-mer oligonucleotides were annealed at 1:1 molar ratio to its complementary oligonucleotides to generate the dsDNA and then radio-labeled with ${ }^{32} \mathrm{P}$ at the $5^{\prime}$-end. GST-ZNF644, GST-ZNF644N300, GST-WIZ, or GST-WIZN200 was incubated with $0.2 \mathrm{nM}$ (molecules) radiolabeled DNA substrates for $2 \mathrm{hr}$ at $4^{\circ} \mathrm{C}$ in buffer D (20 mM HEPES-KOH (pH 7.9), $20 \%$ glycerol (vol/ vol), $0.2 \mathrm{mM}$ EDTA, $0.1 \mathrm{M} \mathrm{KCl}, 0.5 \mathrm{mM}$ PSMF, $1 \mathrm{mM} \mathrm{DTT}$ ) with $1.25 \mu \mathrm{g} / \mu \mathrm{l}$ Bovine serum albumin, $1 \mathrm{mM}$ DTT, $5 \mathrm{mM} \mathrm{MgCl}_{2}$. The samples were resolved by electrophoresis on a $7.5 \%$ polyacrylamide gel in TBE buffer for $70 \mathrm{~min}$ at $60 \mathrm{~V}$. The gel was then dried and exposed to autoradiography film overnight.

\section{Statistical analysis}

In all cases, multiple independent experiments were performed on different days to verify the reproducibility of experimental findings. Two-way comparison was performed using the $t$-test, and ANOVA was used for more than two groups. For all analyses, a $p$ value of less than 0.05 was considered significant. Results are given as means \pm s.d. 


\section{Acknowledgements}

This work was supported by grants from National Institutes of Health (CA132755, CA130899, and CA187209 to XY). XY is a recipient of Era of Hope Scholar Award from the Department of Defense. We thank Sean Lamarche for proofreading.

\section{Additional information}

Funding

\begin{tabular}{lll} 
Funder & Grant reference & Author \\
\hline National Institutes of Health (NIH) & CA132755 & Xiaochun Yu \\
\hline National Institutes of Health (NIH) & CA130899 & Xiaochun Yu \\
\hline National Institutes of Health (NIH) & CA187209 & Xiaochun Yu \\
\hline U.S. Department of Defense & Era of Hope Scholar Award & Xiaochun Yu \\
\hline
\end{tabular}

The funders had no role in study design, data collection and interpretation, or the decision to submit the work for publication.

Author contributions

CB, Acquisition of data, Analysis and interpretation of data, Drafting or revising the article, Contributed unpublished essential data or reagents; $\mathrm{QC}$, Acquisition of data, Analysis and interpretation of data; XY, Conception and design, Analysis and interpretation of data, Drafting or revising the article, Contributed unpublished essential data or reagents

\section{Additional files}

Supplementary file

- Supplementary file 1. PCR primers. All the primers used for RT-PCR and ChIP-qPCR are listed. DOI: 10.7554/eLife.05606.018

Major dataset

The following dataset was generated:

\begin{tabular}{|c|c|c|c|c|}
\hline Author(s) & Year & Dataset title & $\begin{array}{l}\text { Dataset ID } \\
\text { and/or URL }\end{array}$ & $\begin{array}{l}\text { Database, license, and } \\
\text { accessibility information }\end{array}$ \\
\hline $\begin{array}{l}\text { Bian C, Yu } \\
X\end{array}$ & 2014 & $\begin{array}{l}\text { G9a, ZNF644 and WIZ } \\
\text { ChIP-seq results }\end{array}$ & $\begin{array}{l}\text { http://www.ncbi.nlm.nih.gov/ } \\
\text { geo/query/acc.cgi? } \\
\text { acc=GSE62616 }\end{array}$ & $\begin{array}{l}\text { Publicly available at NCBI } \\
\text { Gene Expression Omnibus } \\
\text { (GSE62616). }\end{array}$ \\
\hline
\end{tabular}

\section{References}

Bannister AJ, Zegerman P, Partridge JF, Miska EA, Thomas JO, Allshire RC, Kouzarides T. 2001. Selective recognition of methylated lysine 9 on histone H3 by the HP1 chromo domain. Nature 410:120-124. doi: 10.1038/ 35065138.

Barski A, Cuddapah S, Cui K, Roh TY, Schones DE, Wang Z, Wei G, Chepelev I, Zhao K. 2007. High-resolution profiling of histone methylations in the human genome. Cell 129:823-837. doi: 10.1016/j.cell.2007.05.009.

Bittencourt D, Wu DY, Jeong KW, Gerke DS, Herviou L, lanculescu I, Chodankar R, Siegmund KD, Stallcup MR. 2012. G9a functions as a molecular scaffold for assembly of transcriptional coactivators on a subset of glucocorticoid receptor target genes. Proceedings of the National Academy of Sciences of the United States of America 109:19673-19678. doi: 10.1073/pnas.1211803109.

Chang Y, Sun L, Kokura K, Horton JR, Fukuda M, Espejo A, Izumi V, Koomen JM, Bedford MT, Zhang X, Shinkai Y, Fang J, Cheng X. 2011. MPP8 mediates the interactions between DNA methyltransferase Dnmt3a and H3K9 methyltransferase GLP/G9a. Nature Communications 2:533. doi: 10.1038/ncomms1549.

Chen MW, Hua KT, Kao HJ, Chi CC, Wei LH, Johansson G, Shiah SG, Chen PS, Jeng YM, Cheng TY, Lai TC, Chang JS, Jan YH, Chien MH, Yang CJ, Huang MS, Hsiao M, Kuo ML. 2010. H3K9 histone methyltransferase G9a promotes lung cancer invasion and metastasis by silencing the cell adhesion molecule Ep-CAM. Cancer Research 70:7830-7840. doi: 10.1158/0008-5472.CAN-10-0833.

Chen Q, Chen Y, Bian C, Fujiki R, Yu X. 2013. TET2 promotes histone O-GlcNAcylation during gene transcription. Nature 493:561-564. doi: 10.1038/nature11742. 
Chen X, Skutt-Kakaria K, Davison J, Ou YL, Choi E, Malik P, Loeb K, Wood B, Georges G, Torok-Storb B, Paddison PJ. 2012. G9a/GLP-dependent histone H3K9me2 patterning during human hematopoietic stem cell lineage commitment. Genes \& Development 26:2499-2511. doi: 10.1101/gad.200329.112.

Collins RE, Northrop JP, Horton JR, Lee DY, Zhang X, Stallcup MR, Cheng X. 2008. The ankyrin repeats of G9a and GLP histone methyltransferases are mono- and dimethyllysine binding modules. Nature Structural \& Molecular Biology 15:245-250. doi: 10.1038/nsmb.1384.

Dillon SC, Zhang X, Trievel RC, Cheng X. 2005. The SET-domain protein superfamily: protein lysine methyltransferases. Genome Biology 6:227. doi: 10.1186/gb-2005-6-8-227.

Epsztejn-Litman S, Feldman N, Abu-Remaileh M, Shufaro Y, Gerson A, Ueda J, Deplus R, Fuks F, Shinkai Y, Cedar $H$, Bergman Y. 2008. De novo DNA methylation promoted by G9a prevents reprogramming of embryonically silenced genes. Nature Structural \& Molecular Biology 15:1176-1183. doi: 10.1038/nsmb.1476.

Esteve PO, Chin HG, Smallwood A, Feehery GR, Gangisetty O, Karpf AR, Carey MF, Pradhan S. 2006. Direct interaction between DNMT1 and G9a coordinates DNA and histone methylation during replication. Genes \& Development 20:3089-3103. doi: 10.1101/gad.1463706.

Fang TC, Schaefer U, Mecklenbrauker I, Stienen A, Dewell S, Chen MS, Rioja I, Parravicini V, Prinjha RK, Chandwani R, MacDonald MR, Lee K, Rice CM, Tarakhovsky A. 2012. Histone H3 lysine 9 di-methylation as an epigenetic signature of the interferon response. The Journal of Experimental Medicine 209:661-669. doi: 10.1084/jem. 20112343.

Gyory I, Wu J, Fejer G, Seto E, Wright KL. 2004. PRDI-BF1 recruits the histone H3 methyltransferase G9a in transcriptional silencing. Nature Immunology 5:299-308. doi: 10.1038/ni1046.

Huang J, Dorsey J, Chuikov S, Perez-Burgos L, Zhang X, Jenuwein T, Reinberg D, Berger SL. 2010. G9a and Glp methylate lysine 373 in the tumor suppressor p53. The Journal of Biological Chemistry 285:9636-9641. doi: 10. 1074/jbc.M109.062588.

Klug A, Rhodes D. 1987. Zinc fingers: a novel protein fold for nucleic acid recognition. Cold Spring Harbor Symposia on Quantitative Biology 52:473-482. doi: 10.1101/SQB.1987.052.01.054.

Kubicek S, O'Sullivan RJ, August EM, Hickey ER, Zhang Q, Teodoro ML, Rea S, Mechtler K, Kowalski JA, Homon CA, Kelly TA, Jenuwein T. 2007. Reversal of H3K9me2 by a small-molecule inhibitor for the G9a histone methyltransferase. Molecular Cell 25:473-481. doi: 10.1016/j.molcel.2007.01.017.

Lachner M, O'Carroll D, Rea S, Mechtler K, Jenuwein T. 2001. Methylation of histone H3 lysine 9 creates a binding site for HP1 proteins. Nature 410:116-120. doi: 10.1038/35065132.

Lee DY, Northrop JP, Kuo MH, Stallcup MR. 2006. Histone H3 lysine 9 methyltransferase G9a is a transcriptional coactivator for nuclear receptors. The Journal of Biological Chemistry 281:8476-8485. doi: 10.1074/jbc. M511093200.

Mozzetta C, Pontis J, Fritsch L, Robin P, Portoso M, Proux C, Margueron R, Ait-Si-Ali S. 2014. The histone H3 lysine 9 methyltransferases G9a and GLP regulate polycomb repressive complex 2-mediated gene silencing. Molecular Cell 53:277-289. doi: 10.1016/j.molcel.2013.12.005.

Nielsen AL, Oulad-Abdelghani M, Ortiz JA, Remboutsika E, Chambon P, Losson R. 2001. Heterochromatin formation in mammalian cells: interaction between histones and HP1 proteins. Molecular Cell 7:729-739. doi: 10. 1016/S1097-2765(01)00218-0.

Nishio H, Walsh MJ. 2004. CCAAT displacement protein/cut homolog recruits G9a histone lysine methyltransferase to repress transcription. Proceedings of the National Academy of Sciences of the United States of America 101:11257-11262. doi: 10.1073/pnas.0401343101.

Ogawa H, Ishiguro K, Gaubatz S, Livingston DM, Nakatani Y. 2002. A complex with chromatin modifiers that occupies E2F- and Myc-responsive genes in G0 cells. Science 296:1132-1136. doi: 10.1126/science.1069861.

Purcell DJ, Jeong KW, Bittencourt D, Gerke DS, Stallcup MR. 2011. A distinct mechanism for coactivator versus corepressor function by histone methyltransferase G9a in transcriptional regulation. The Journal of Biological Chemistry 286:41963-41971. doi: 10.1074/jbc.M111.298463.

Roopra A, Qazi R, Schoenike B, Daley TJ, Morrison JF. 2004. Localized domains of G9a-mediated histone methylation are required for silencing of neuronal genes. Molecular Cell 14:727-738. doi: 10.1016/j.molcel.2004. 05.026 .

Schaefer A, Sampath SC, Intrator A, Min A, Gertler TS, Surmeier DJ, Tarakhovsky A, Greengard P. 2009. Control of cognition and adaptive behavior by the GLP/G9a epigenetic suppressor complex. Neuron 64:678-691. doi: 10. 1016/j.neuron.2009.11.019.

Shaffer AL, Lin KI, Kuo TC, Yu X, Hurt EM, Rosenwald A, Giltnane JM, Yang L, Zhao H, Calame K, Staudt LM. 2002. Blimp-1 orchestrates plasma cell differentiation by extinguishing the mature B cell gene expression program. Immunity 17:51-62. doi: 10.1016/S1074-7613(02)00335-7.

Shinkai Y, Tachibana M. 2011. H3K9 methyltransferase G9a and the related molecule GLP. Genes \& Development 25:781-788. doi: 10.1101/gad.2027411.

Su RC, Brown KE, Saaber S, Fisher AG, Merkenschlager M, Smale ST. 2004. Dynamic assembly of silent chromatin during thymocyte maturation. Nature Genetics 36:502-506. doi: 10.1038/ng1351.

Tachibana M, Matsumura Y, Fukuda M, Kimura H, Shinkai Y. 2008. G9a/GLP complexes independently mediate H3K9 and DNA methylation to silence transcription. The EMBO Journal 27:2681-2690. doi: 10.1038/emboj.2008. 192.

Tachibana M, Nozaki M, Takeda N, Shinkai Y. 2007. Functional dynamics of H3K9 methylation during meiotic prophase progression. The EMBO Journal 26:3346-3359. doi: 10.1038/sj.emboj.7601767. 
Tachibana M, Sugimoto K, Fukushima T, Shinkai Y. 2001. Set domain-containing protein, G9a, is a novel lysinepreferring mammalian histone methyltransferase with hyperactivity and specific selectivity to lysines 9 and 27 of histone H3. The Journal of Biological Chemistry 276:25309-25317. doi: 10.1074/jbc.M101914200.

Tachibana M, Sugimoto K, Nozaki M, Ueda J, Ohta T, Ohki M, Fukuda M, Takeda N, Niida H, Kato H, Shinkai Y. 2002. G9a histone methyltransferase plays a dominant role in euchromatic histone H3 lysine 9 methylation and is essential for early embryogenesis. Genes \& Development 16:1779-1791. doi: 10.1101/gad.989402.

Tachibana M, Ueda J, Fukuda M, Takeda N, Ohta T, Iwanari H, Sakihama T, Kodama T, Hamakubo T, Shinkai Y. 2005. Histone methyltransferases G9a and GLP form heteromeric complexes and are both crucial for methylation of euchromatin at H3-K9. Genes \& Development 19:815-826. doi: 10.1101/gad.1284005.

Ueda J, Tachibana M, Ikura T, Shinkai Y. 2006. Zinc finger protein Wiz links G9a/GLP histone methyltransferases to the co-repressor molecule CtBP. The Journal of Biological Chemistry 281:20120-20128. doi: 10.1074/jbc. M603087200.

Wen B, Wu H, Shinkai Y, Irizarry RA, Feinberg AP. 2009. Large histone H3 lysine 9 dimethylated chromatin blocks distinguish differentiated from embryonic stem cells. Nature Genetics 41:246-250. doi: 10.1038/ng.297.

Ye T, Krebs AR, Choukrallah MA, Keime C, Plewniak F, Davidson I, Tora L. 2011. seqMINER: an integrated ChIPseq data interpretation platform. Nucleic Acids Research 39:e35. doi: 10.1093/nar/gkq1287.

Zhang F, Ma J, Wu J, Ye L, Cai H, Xia B, Yu X. 2009. PALB2 links BRCA1 and BRCA2 in the DNA-damage response. Current Biology 19:524-529. doi: 10.1016/j.cub.2009.02.018. 